\title{
Cut-off values for positive bronchodilator response in healthy Thai preschool children using forced oscillation technique
}

\author{
Kanokporn Udomittipong, ${ }^{1}$ Jurairut Triwatanawong, ${ }^{1}$ Akarin Nimmannit, ${ }^{2}$ Chulaluk Komoltri ${ }^{3}$
}

\begin{abstract}
Background: Forced oscillation technique (FOT) requires minimal patient cooperation and is particularly useful in young children. Bronchodilator test is a valuable tool for wheezy and asthmatic patients. The cut-off value for bronchodilator response by FOT in healthy Thai children has not been reported.
\end{abstract}

Objective: To determine the cut-off values for positive bronchodilator response in healthy Thai preschool children using pseudorandom FOT.

Methods: FOT was used to measure respiratory function at baseline and after $400 \mathrm{mcg}$ MDI salbutamol in healthy Thai children aged 3-6 years. Respiratory resistance (Rrs) and reactance (Xrs) at 6, 8, and $10 \mathrm{~Hz}$ were collected. Pre- and post-bronchodilator tests were compared using paired t-test. Absolute and percent changes after bronchodilator were calculated and their cut-off values were defined as mean $\pm 1.96 \mathrm{SD}$. Correlation between each of those and baseline data was analyzed using Pearson's correlation coefficient.

Results: Of the 150 enrolled children, FOT measurement at baseline and after bronchodilator was successfully completed in 111 children (51 boys). The mean \pm standard deviation age, height, and arm span was $5.2 \pm 1.1$ years, $109.3 \pm 8.7$ $\mathrm{cm}$, and $107.2 \pm 9.1 \mathrm{~cm}$, respectively. No correlation was observed between any absolute or percent changes in bronchodilator response and gender, age, height, or arm span. The cut-off values established for bronchodilator response by percent change were, as follows: Rrs6: $-23 \%$, Rrs8: -20\%, Rrs10: -20\%, Xrs6: 36\%, Xrs8: 60\%, and Xrs10: $43 \%$.

Conclusions: The cut-off values identified in this study will be useful for evaluating bronchodilator response by FOT in wheezy and asthmatic young children.

Key words: cut-off values, bronchodilator response, preschool children, forced oscillation, pulmonary function test

\section{From:}

Division of Pulmonology,Department of Pediatrics,

Faculty of Medicine Siriraj Hospital, Mahidol University, Bangkok, Thailand

Office for Research and Development, Faculty of Medicine Siriraj Hospital, Mahidol University, Bangkok, Thailand

Division of Clinical Epidemiology, Department of Research and Development, Faculty of Medicine Siriraj Hospital,

Mahidol University, Bangkok, Thailand

\section{Introduction}

Pulmonary function test and bronchodilator test are commonly used in clinical practice to diagnose and assess patients with respiratory problems. Conventional spirometry is an effort-dependent test that is used mostly in adults and older children. It is difficult to achieve accurate spirometry measurements in young children due to their shorter attention span and their limited ability to clearly understand instructions and cooperate.
Corresponding author:

Kanokporn Udomittipong

Associate Professor of Pediatrics

Division of Pulmonology,Department of Pediatrics

Faculty of Medicine Siriraj Hospital, Mahidol University

2 Wanglang Road, Bangkoknoi, Bangkok 10700, Thailand

E-mail: kanokporn.udo@mahidol.ac.th

Forced oscillation technique (FOT) is a pulmonary function testing method that can be successfully performed in preschool children with the success rate of $80-90 \%{ }^{1-5}$ and this technique is used with increasing frequency in lung function laboratories. FOT requires minimal patient cooperation with tidal breathing to measure lung function, which makes it suitable for use in young children. The oscillatory signal delivered by the machine is superimposed on spontaneous breathing, 
which facilitates measurement of the mechanical properties of the respiratory system. FOT variable outcome is respiratory impedance (Zrs), which comprises respiratory resistance (Rrs) and respiratory reactance (Xrs). ${ }^{6}$

Asthma and wheezing is very common among preschool children, and there are various phenotypes of wheezing in this age group. Bronchodilator test is an effective method for assessing bronchial hyperreactivity. Several previous studies reported FOT to be a valid tool for assessment of bronchodilator response in asthmatic and wheezy children. ${ }^{7-11}$ However, before FOT can be integrated into clinical practice to define bronchial hyperreactivity, data from healthy population and asthmatic children are required.

Given that lung function can vary by ethnicity, ${ }^{12-14}$ the cutoff values used in clinical practice should be derived from the ethnic group predominantly treated in routine clinical practice. ${ }^{15,16}$ Most of the studies in bronchodilator testing by commercially available pseudorandom FOT in healthy preschool children were conducted in Caucasian populations and reported the cut-off percent changes for Rrs ranging from -30\% to $-40 \%$ and $50 \%$ to $70 \%$ for $\mathrm{Xrs}^{4,9,17}$ Our review of the literature revealed no reports in Thai children, and only limited data from Asian population. ${ }^{3}$

Accordingly, the aim of this study was to determine the cut-off values for positive bronchodilator response in healthy Thai preschool children using pseudorandom forced oscillation technique. Our intent was to improve the evaluation and management of asthmatic and wheezy children, and to improve our understanding of wheezing phenotypes.

\section{Methods \\ Study Population}

This study recruited healthy children aged 3-6 years from kindergartens and primary schools in Bangkok, the capital city of Thailand, and the surrounding area during the April 2014 to December 2016 study period. This study was conducted at the Division of Pulmonology, Department of Pediatrics, Siriraj Hospital - Thailand's largest national tertiary referral center. The exclusion criteria were children with neuromuscular diseases, heart diseases, and/or chronic lung diseases, such as bronchopulmonary dysplasia and asthma from a past history taking. Children with the past history of doctor-diagnosed or parentally-reported wheezing, asthma, or respiratory distress at any time of their life were excluded. We also ex-cluded children with history of pneumonia, wheezing, respiratory distress, preterm birth, birth weight $<2,500$ $g$, respiratory tract infection within 2 weeks before the study, and exposure to environmental tobacco smoke. Baseline data including age, weight, height, arm span, and gender were recorded. Written informed consent was obtained from a parent or legal guardian of each participating child before inclusion. This study was approved by the Siriraj Institutional Review Board (SIRB), Faculty of Medicine Siriraj Hospital, Mahidol University, Bangkok, Thailand (COA no. 052/2014), and complied with the principles set forth in the Declaration of Helsinki (1964) and all of its subsequent amendments.

\section{Forced oscillation technique (FOT) measurement}

The commercially available Quark i2m forced oscillation system (Cosmed srl, Rome, Italy) was used to obtain respiratory impedance (Zrs), which is made up of both respiratory resistance (Rrs) and reactance (Xrs). The equipment generates a pseudorandom noise with multiple frequencies between 4-48 Hz, and a measurement period of 8 seconds. Pseudorandom noise is composite signals that are applied to spontaneous breathing. Coherence function, which is a measure of the linear relationship between input and output signals and reflects the validity of the measurements at individual frequencies, is automatically assessed at each frequency. ${ }^{6,18}$

Respiratory impedance was measured according to American Thoracic Society/European Respiratory Society guidelines (ATS/ERS). ${ }^{19}$ Measurements were performed with children in a sitting position and their head in a neutral position with a nose clip in place. The cheeks and lower jaw of each child were supported by the hands of the respiratory technician. Children breathed normally through a mouth piece fitted with an antibacterial filter (model: A 182300 044; Cosmed), which was connected to the forced oscillation system. ${ }^{6,19}$ Three to five technically acceptable measurements were obtained. Once the baseline measurements were recorded, $400 \mu \mathrm{g}$ of inhaled salbutamol (Ventolin ${ }^{\circledast}$ MDI $100 \mu \mathrm{g} /$ dose; GlaxoSmithKline, Brentford, United Kingdom) was given at tidal breath via valve holding chamber (AeroChamber Plus; Trudell Medical International, London, Ontario, Canada). Post-bronchodilator measurement was performed at 15-20 minutes following bronchodilator administration.

Measurements were considered acceptable if coherence at an individual frequency was $\geq 0.95$, and excluded if $\geq 3$ individual frequencies had a coherence of $<0.95$. Measurements with swallowing, mouth leak, mouth movement, glottic closure, or audible noise were excluded. Respiratory resistance and reactance at 6,8 , and $10 \mathrm{~Hz}$ were reported and analyzed. These parameters were coded, as follows: Rrs6, Rrs8, and Rrs10 for resistance at 6,8 , and $10 \mathrm{~Hz}$, and Xrs6, Xrs8, and Xrs10 for reactance at 6,8 , and $10 \mathrm{~Hz}$.

\section{Statistical analysis}

All data analyses were performed using SPSS for Windows version 19 (SPSS, Inc., Chicago, IL, USA). Data are expressed as mean \pm standard deviation (SD). Student's paired $t$-test was used to compare between pre- and post-bronchodilator Rsr and Xrs values. Absolute and percent changes after bronchodilator for all Rrs and Xrs parameters were calculated. Cut-off values for positive bronchodilator response were defined by mean $\pm 1.96 \mathrm{SD}$ of absolute and percent changes. Correlation between post-bronchodilator change in Rrs and Xrs values and baseline data, including age, height, arm span, and baseline measurement, were assessed by Pearson's correlation coefficient. A two-sided $p$-value less than 0.05 was considered to be statistically significant. 


\section{Results}

FOT was attempted in 150 children and measurement was successfully completed for both baseline measurement and bronchodilator test in $111(74 \%)$ of them. The gender proportion breakdown was 51 (45.9\%) boys and 60 (54.1\%) girls. The mean \pm standard deviation age, height, weight, and arm span was $5.2 \pm 1.1$ years, $109.3 \pm 8.7 \mathrm{~cm}, 18.8 \pm 4.1 \mathrm{~kg}$, and $107.2 \pm 9.1 \mathrm{~cm}$, respectively. Baseline Rrs and Xrs values are shown in Table 1.

\section{Post-bronchodilator data}

Statistically significant difference was observed between pre- and post-bronchodilator values for all Rrs and Xrs parameters in both absolute change and percent change analyses. The cut-off values for bronchodilator response for absolute (percent) change in Rrs (defined as the lower limit of the mean change $\pm 1.96 \mathrm{SD})$ were $-1.13(-23 \%),-0.92(-20 \%)$, and $-0.94(-20 \%)$ for Rrs6, Rrs8, and Rrs10, respectively. The cut-off values for Xrs6, Xrs8, and Xrs10 (defined as the upper

Table 1. Post-bronchodilator change in respiratory impedance

\begin{tabular}{|c|c|c|c|c|c|c|}
\hline & Rrs6 & Rrs8 & Rrs10 & Xrs6 & Xrs8 & Xrs10 \\
\hline $\begin{array}{l}\text { Baseline } \\
\text { (hPasec/L), mean } \pm \text { SD }\end{array}$ & $4.85 \pm 0.52$ & $4.52 \pm 0.44$ & $4.56 \pm 0.47$ & $-0.88 \pm 0.21$ & $-0.65 \pm 0.23$ & $-0.70 \pm 0.23$ \\
\hline $\begin{array}{l}\text { Post-BD } \\
(\text { hPasec/L), mean } \pm \text { SD }\end{array}$ & $4.55 \pm 0.48$ & $4.25 \pm 0.45$ & $4.27 \pm 0.47$ & $-0.80 \pm 0.21$ & $-0.61 \pm 0.21$ & $-0.61 \pm 0.21$ \\
\hline \multicolumn{7}{|l|}{ Absolute change after $\mathrm{BD}^{\mathrm{a}}$} \\
\hline - Mean \pm SD $($ hPasec/L $)$ & $-0.31 \pm 0.42$ & $-0.27 \pm 0.33$ & $-0.29 \pm 0.33$ & $0.08 \pm 0.19$ & $0.04 \pm 0.21$ & $0.09 \pm 0.19$ \\
\hline - Normal range (hPasec/L) & -1.13 to 0.51 & -0.92 to 0.38 & -0.94 to 0.36 & -0.29 to 0.45 & -0.37 to 0.45 & -0.28 to 0.41 \\
\hline - $P$-value & $<0.001$ & $<0.001$ & $<0.001$ & $<0.001$ & 0.039 & $<0.001$ \\
\hline \multicolumn{7}{|l|}{$\%$ change after $\mathrm{BD}^{\mathrm{b}}$} \\
\hline - Mean \pm SD (\%) & $-5.91 \pm 8.74$ & $-5.84 \pm 7.20$ & $-6.07 \pm 7.14$ & $-9.01 \pm 23.11$ & $-6.05 \pm 33.91$ & $-12.46 \pm 28.14$ \\
\hline - Normal range (\%) & -23.04 to 11.22 & -19.95 to 8.27 & -20.06 to 7.92 & -54.31 to 36.30 & -72.51 to 60.41 & -67.60 to 42.79 \\
\hline - $P$-value & $<0.001$ & $<0.001$ & $<0.001$ & 0.003 & 0.042 & 0.001 \\
\hline
\end{tabular}

A $p$-value $<0.05$ indicates statistical significance

Normal range $=$ mean $\pm 1.96 \mathrm{SD}$

a Absolute change $=$ postbronchodilator - prebronchodilator

b $\%$ change $=($ postbronchodilator - prebronchodilator $) * 100 /$ prebronchodilator

Abbreviations: Rrs, respiratory resistance; Xrs, respiratory reactance; BD, bronchodilator; SD, standard deviation
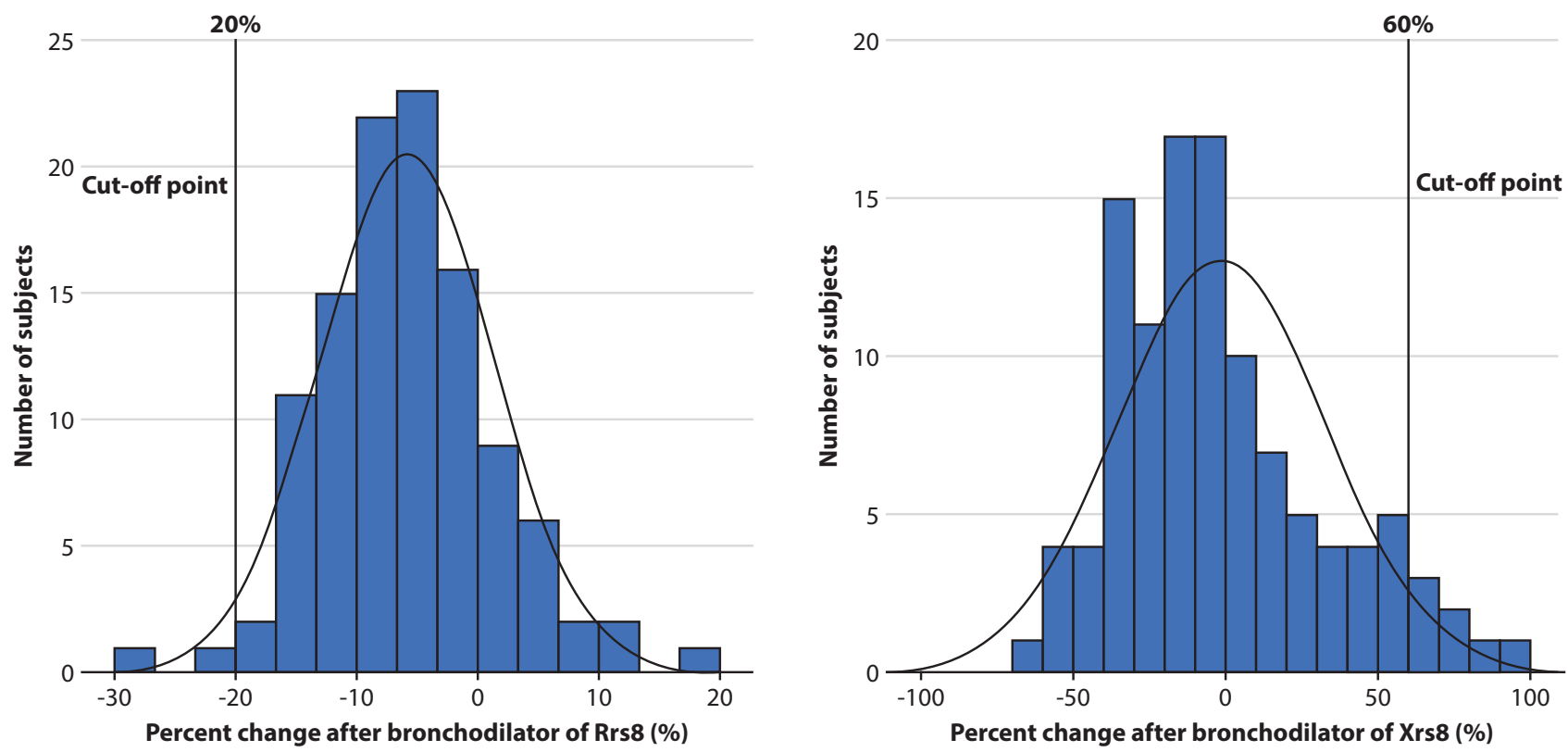

Figure 1. Histogram of percent change after bronchodilator in Rrs8 and Xrs8 and their cut-off value 
limit of the mean change \pm 1.96 SD) were 0.45 (36\%), 0.45 (60\%), and $0.41(43 \%)$, respectively (Table 1). The data of percent change after bronchodilator of Rrs and Xrs at 6, 8, and 10 $\mathrm{Hz}$ demonstrated normal distribution. Histograms of percent change in Rrs8 and Xrs8 and their cut-off value were demonstrated in Figure 1.
Correlation between post-bronchodilator change in Rrs and Xrs and gender, age, height, arm span, and baseline measurement

This study found no correlation between absolute and percent change after bronchodilator for any Rrs or Xrs value and gender, age, height, or arm span. (Tables 2, 3, and 4).

Table 2. Comparison in mean percent and absolute change in Rrs and Xrs variables after bronchodilator between boys and girls

\begin{tabular}{|c|c|c|c|c|c|c|}
\hline & \multicolumn{3}{|c|}{ Percent change (\%) } & \multicolumn{3}{|c|}{ Absolute change (hPasec/L) } \\
\hline & \multicolumn{2}{|c|}{ mean \pm SD } & \multirow{2}{*}{ p-value } & \multicolumn{2}{|c|}{ mean \pm SD } & \multirow{2}{*}{ p-value } \\
\hline & Boy & Girl & & Boy & Girl & \\
\hline$\Delta$ Rrs6 & $-7.70 \pm 9.23$ & $-4.39 \pm 8.07$ & 0.06 & $-0.39 \pm 0.45$ & $-0.25 \pm 0.38$ & 0.06 \\
\hline$\Delta$ Rrs8 & $-7.11 \pm 7.97$ & $-4.77 \pm 6.36$ & 0.09 & $-0.33 \pm 0.36$ & $-0.28 \pm 0.29$ & 0.08 \\
\hline$\Delta \operatorname{Rrs10}$ & $-7.25 \pm 7.85$ & $-5.06 \pm 6.36$ & 0.11 & $-0.34 \pm 0.35$ & $-0.24 \pm 0.30$ & 0.11 \\
\hline$\Delta \mathrm{Xrs6}$ & $-9.32 \pm 25.19$ & $-4.28 \pm 21.12$ & 0.25 & $0.09 \pm 0.21$ & $0.06 \pm 0.18$ & 0.36 \\
\hline$\Delta \mathrm{Xrs} 8$ & $-9.41 \pm 32.40$ & $-4.96 \pm 34.03$ & 0.20 & $0.09 \pm 0.23$ & $0.05 \pm 0.17$ & 0.25 \\
\hline$\Delta \mathrm{Xrs} 10$ & $-15.97 \pm 24.13$ & $-10.74 \pm 30.21$ & 0.30 & $0.14 \pm 0.19$ & $0.09 \pm 0.17$ & 0.28 \\
\hline
\end{tabular}

A $p$-value $<0.05$ indicates statistical significance

Abbreviations: Rrs, respiratory resistance; Xrs, respiratory reactance; $\mathrm{SD}$, standard deviation; $\Delta$, change

Table 3. Correlation between percent change in Rrs and Xrs variables and age, height, arm span, and baseline measurement

\begin{tabular}{|c|c|c|c|c|c|c|c|c|}
\hline & \multicolumn{2}{|c|}{ Age (years) } & \multicolumn{2}{|c|}{ Height (cm) } & \multicolumn{2}{|c|}{ Arm span (cm) } & \multicolumn{2}{|c|}{ Baseline (hPasec/L) } \\
\hline & $r$ & $p$ & $\boldsymbol{r}$ & $p$ & $r$ & $p$ & $r$ & $p$ \\
\hline$\% \Delta \operatorname{Rrs6}$ & 0.10 & 0.28 & 0.13 & 0.19 & 0.08 & 0.42 & -0.43 & $<0.001$ \\
\hline$\% \Delta \operatorname{Rrs} 8$ & 0.01 & 0.91 & 0.04 & 0.71 & 0.01 & 0.91 & -0.28 & 0.003 \\
\hline$\% \Delta \operatorname{Rrs10}$ & -0.01 & 0.91 & 0.02 & 0.83 & 0.01 & 0.84 & -0.26 & 0.007 \\
\hline$\% \Delta \mathrm{Xrs} 6$ & 0.12 & 0.22 & 0.05 & 0.59 & 0.06 & 0.56 & 0.38 & $<0.001$ \\
\hline$\% \Delta \mathrm{Xrs} 8$ & 0.06 & 0.55 & 0.03 & 0.76 & -0.02 & 0.87 & 0.48 & $<0.001$ \\
\hline$\% \Delta \mathrm{Xrs} 10$ & 0.02 & 0.88 & 0.01 & 0.90 & -0.02 & 0.84 & 0.35 & $<0.001$ \\
\hline
\end{tabular}

A $p$-value $<0.05$ indicates statistical significance

Abbreviations: Rrs, respiratory resistance; Xrs, respiratory reactance; $r$, Pearson's correlation coefficient; $p$, $\mathrm{p}$-value; $\Delta$, change

Table 4. Correlation between absolute change in Rrs and Xrs variables and age, height, arm span, and baseline measurement

\begin{tabular}{|c|c|c|c|c|c|c|c|c|}
\hline & \multicolumn{2}{|c|}{ Age (years) } & \multicolumn{2}{|c|}{ Height (cm) } & \multicolumn{2}{|c|}{ Arm span (cm) } & \multicolumn{2}{|c|}{ Baseline (hPasec/L) } \\
\hline & $r$ & $p$ & $r$ & $p$ & $r$ & $p$ & $r$ & $p$ \\
\hline$\Delta$ Rrs6 & 0.15 & 0.13 & 0.17 & 0.08 & 0.12 & 0.19 & -0.51 & $<0.001$ \\
\hline$\Delta$ Rrs8 & 0.05 & 0.59 & 0.08 & 0.41 & 0.06 & 0.55 & -0.40 & $<0.001$ \\
\hline$\Delta \operatorname{Rrs} 10$ & 0.04 & 0.66 & 0.08 & 0.43 & 0.04 & 0.70 & -0.35 & $<0.001$ \\
\hline$\Delta \mathrm{Xrs6}$ & -0.16 & 0.09 & -0.10 & 0.30 & -0.10 & 0.31 & -0.48 & $<0.001$ \\
\hline$\Delta \mathrm{Xrs} 8$ & -0.05 & 0.59 & 0.01 & 0.98 & 0.05 & 0.62 & -0.54 & $<0.001$ \\
\hline$\Delta \mathrm{Xrs} 10$ & -0.06 & 0.56 & -0.02 & 0.84 & 0.01 & 0.95 & -0.50 & $<0.001$ \\
\hline
\end{tabular}

A $p$-value $<0.05$ indicates statistical significance

Abbreviations: Rrs, respiratory resistance; Xrs, respiratory reactance; $r$, Pearson's correlation coefficient; $p$, $\mathrm{p}$-value; $\Delta$, change 
The absolute and percent changes in all Rrs and Xrs values were related to their corresponding baseline measurement, but the coefficient of correlation ( $r$ ) values for percent changes were lower than those for absolute changes (Tables 3 and 4).

\section{Discussion}

Consistent with the findings of several previous studies, ${ }^{3,9,17,20,21}$ this study found statistically significant differences for all Rrs and Xrs parameters between pre- and post-bronchodilator administration in both absolute and percent change among healthy Thai preschool children.

In order to introduce bronchodilator test by FOT into clinical practice, correlations between bronchodilator response and age, gender, height, and baseline lung function should be evaluated. If any significant associations are found, the related factors must be taken into account when interpreting the result. ${ }^{22}$ Consistent with the findings of $\mathrm{Vu}$, et al., ${ }^{3}$ we found the absolute and percent changes in FOT variables after bronchodilator administration to be independent of gender, age, and height. Thamrin, et al. ${ }^{9}$ also reported no correlation between height and bronchodilator response, as expressed by either absolute change or percent change. This finding supports that change values in Rrs and Xrs after bronchodilator can be used to interpret bronchial hyperresponsiveness irrespective of these factors.

Our study found both absolute and percent changes in bronchodilator response to be significantly related to their corresponding baseline values; however, percent changes had lower correlation coefficient, which is consistent with the findings of Calogero, et al. ${ }^{4,17}$ and Thamrin, et al. ${ }^{9}$ Accordingly, our finding supports percent change values as more appropriate than absolute change values for interpreting bronchial hyper-responsiveness as recommended by Thamrin, et al. ${ }^{9}$

The cut-off values that we established in this study for bronchodilator response by percent change were, as follows: $-23 \%$ for Rrs6, $-20 \%$ for Rrs8, $-20 \%$ for Rrs 10, 36\% for Xrs6, $60 \%$ for Xrs 8 , and $43 \%$ for Xrs 10 . There are a relatively small number of reports on reference values that define bronchodilator response by pseudorandom FOT. Previously reported cut-off percent changes for Rrs ranged from $-30 \%$ to$40 \%,{ }^{3,4,9,17} 50 \%$ to $70 \%$ for $\mathrm{Xrs},{ }^{4,9,17}$ and $16 \%$ for $\mathrm{Xrs} 8$ by $\mathrm{Vu}$, et $a .^{3}$ Previous studies by Hellinckx, et al. ${ }^{20}$ and Malmberg, et $a .^{21}$ in preschool children using impulse oscillometry technique proposed cut-offs for Rrs5 of $-41 \%$ and $-37 \%$, respectively. While, Nielsen, et al. ${ }^{23}$ reported a lower Rrs5 value of $-28 \%$. Our cut-off values for Rrs $(-20 \%$ to $-30 \%)$ are lower than those reported in most previous studies, but they are comparable with that values reported by Nielsen, et al. ${ }^{23}$ The differences between studies are likely due to differences in the ethnicity of the study population and/or differences in equipment specifications. In addition, the different results may be affected by techniques and children's co-operations. Our measurement technique is less likely to be the cause of this difference, as we followed standardized protocol according to ATS/ ERS recommendation. ${ }^{19}$ This result supports the assertion that lung function interpretation should be based on ethnicity-specific data. ${ }^{15,16}$
The strength of our study is that arm span was included with other demographic and anthropometric variables, and the result of no significant association with changes after bronchodilator was the same for all basic patient data. As a result, patients in whom arm span is used instead of height to predict lung function can use the same Rrs and Xrs cut-off values for bronchodilator response. Another strength is that we analyzed association between changes after bronchodilator and baseline lung function to evaluate how to suitably express bronchodilator response in terms of absolute or percent change. Lastly, given that no cut-off values to define bronchodilator response by FOT in Thai preschool children have been reported and studies in Asian population are scarce, our data will provide clinical and/or comparative benefit to physicians in Thailand, in Asia, and to pediatric pulmonologists worldwide.

There is no confounding factor in this study because it is not a study to determine risk factors of a certain disease. Regarding selection bias, healthy preschool children from Bangkok and surrounding areas were recruited. We were not able to do a multistage cluster sampling in order to get a good representative of all healthy preschool children in Thailand. For measurement errors, we try to minimize this limitation. The pulmonary function testing was performed by only two experience technicians and the same equipment and standard technique was used to obtain the measurement. Rrx and Xrs values were averaged from 3-5 technically acceptable measurements. Another mentionable limitation is that while we intend our findings will have benefit for pulmonary clinicians in other Asian countries, we cannot be certain that these findings can be extrapolated to preschool children from other countries in Asia.

\section{Conclusions}

The present study advocates using percentage change between baseline and post-bronchodilator to determine bronchodilator response. The cut-off values for evaluating bronchodilator response by FOT in young Thai children are, as follows: Rrs6: $-23 \%$, Rrs8: $-20 \%$, and, Rrs10: $-20 \%$; and, Xrs6: 36\%, Xrs8: $60 \%$, and Xrs10: 43\%. No significant association was observed between any demographic or anthropometric parameters and absolute or percent changes in bronchodilator response. The cut-off values identified in this study will be useful for evaluating bronchodilator response by FOT in wheezy and asthmatic young Thai children.

\section{Acknowledgements}

The authors gratefully acknowledge the children and parents that generously agreed to participate in this study. The authors would also like to thank Mr. Kevin P. Jones for editing the manuscript.

\section{Author Disclosure Statement}

All authors declare no personal or professional conflicts of interest, and no financial support from the companies that produce and/or distribute the drugs, devices, or materials described in this report. 


\section{Funding}

This research project was supported by a grant from the Siriraj Routine to Research Management Fund, Faculty of Medicine Siriraj Hospital, Mahidol University (grant no. R2R. 264/14).

\section{Authors' contributions}

- Udomittipong K. contributed to study conception, study design, statistical analysis, and manuscript preparation.

- Triwatanawong J. recruited study participants, conducted the fieldwork, performed data collection.

- Nimmannit A. contributed to study conception and design.

- Komoltri C. contributed to study design, statistical analysis, and interpretation of data.

\section{References}

1. Hall GL, Sly PD, Fukushima T, Kusel MM, Franklin PJ, Horak F, Jr., et al. Respiratory function in healthy young children using forced oscillations. Thorax. 2007;62(6):521-6.

2. Udomittipong K, Sly PD, Patterson HJ, Gangell CL, Stick SM, Hall GL. Forced oscillations in the clinical setting in young children with neonatal lung disease. Eur Respir J. 2008;31(6):1292-9.

3. Vu LT, Demoulin B, Nguyen YT, Nguyen MT, Marchal F. Respiratory impedance and response to salbutamol in healthy Vietnamese children. Pediatr Pulmonol. 2008;43(10):1013-9.

4. Calogero C, Parri N, Baccini A, Cuomo B, Palumbo M, Novembre E, et al. Respiratory impedance and bronchodilator response in healthy Italian preschool children. Pediatr Pulmonol. 2010;45(11):1086-94.

5. Udomittipong K. Respiratory impedance reference values for forced oscillation technique predicted by arm span and height in Thai preschool children. Pediatric allergy, immunology, and pulmonology. 2017;30: 97-102.

6. Hall G, Brookes I. Techniques for the measurement of lung function in toddlers and preschool children. In: Hammer J, Eber E, eds, editors. Paediatric pulmonary function testing. Basel: Karger; 2005. p. 66-77.

7. Delacourt C, Lorino H, Herve-Guillot M, Reinert P, Harf A, Housset B. Use of the forced oscillation technique to assess airway obstruction and reversibility in children. Am J Respir Crit Care Med. 2000;161(3 Pt 1):730-6.

8. Lall CA, Cheng N, Hernandez P, Pianosi PT, Dali Z, Abouzied A, et al Airway resistance variability and response to bronchodilator in children with asthma. Eur Respir J. 2007;30(2):260-8.
9. Thamrin C, Gangell CL, Udomittipong $\mathrm{K}$, Kusel $\mathrm{MM}$, Patterson $\mathrm{H}$, Fukushima T, et al. Assessment of bronchodilator responsiveness in preschool children using forced oscillations. Thorax. 2007;62(9):814-9.

10. Oostveen E, Dom S, Desager K, Hagendorens M, De Backer W, Weyler J. Lung function and bronchodilator response in 4-year-old children with different wheezing phenotypes. Eur Respir J. 2010;35(4):865-72.

11. Vu LT, Demoulin B, Nguyen MT, Nguyen YT, Marchal F. Respiratory impedance and response to salbutamol in asthmatic Vietnamese children. Pediatr Pulmonol. 2010;45(4):380-6.

12. Pool JB, Greenough A. Ethnic variation in respiratory function in young children. Respir Med. 1989;83(2):123-5

13. Lum S, Aurora P. Does ethnicity influence lung function in preschool children? Expert Rev Respir Med. 2010;4(3):267-9.

14. Quanjer PH. Lung function, race and ethnicity: a conundrum. Eur Respir J. 2013;41(6):1249-51.

15. Pellegrino R, Viegi G, Brusasco V, Crapo RO, Burgos F, Casaburi $\mathrm{R}$, et al. Interpretative strategies for lung function tests. Eur Respir J. 2005; 26(5):948-68

16. Stanojevic S, Wade A, Stocks J. Reference values for lung function: past, present and future. Eur Respir J. 2010;36(1):12-9.

17. Calogero C, Simpson SJ, Lombardi E, Parri N, Cuomo B, Palumbo M, et al. Respiratory impedance and bronchodilator responsiveness in healthy children aged 2-13 years. Pediatr Pulmonol. 2013;48(7):707-15.

18. Daroczy B, Hantos Z. Generation of optimum pseudorandom signals for respiratory impedance measurements. Int J Biomed Comput. 1990; 25(1):21-31.

19. Beydon N, Davis SD, Lombardi E, Allen JL, Arets HG, Aurora P, et al An official American Thoracic Society/European Respiratory Society statement: pulmonary function testing in preschool children. Am J Respir Crit Care Med. 2007;175(12):1304-45.

20. Hellinckx J, De Boeck K, Bande-Knops J, van der Poel M, Demedts M. Bronchodilator response in 3-6.5 years old healthy and stable asthmatic children. Eur Respir J. 1998;12(2):438-43.

21. Malmberg LP, Pelkonen A, Poussa T, Pohianpalo A, Haahtela T, Turpeinen M. Determinants of respiratory system input impedance and bronchodilator response in healthy Finnish preschool children. Clin Physiol Funct Imaging. 2002;22(1):64-71.

22. Thamrin C, Gangell CL, Kusel MM, Schultz A, Hall GL, Stick SM, et al. Expression of bronchodilator response using forced oscillation technique measurements: absolute versus relative. Eur Respir J. 2010;36(1):212; author reply 3 .

23. Nielsen KG, Bisgaard H. Discriminative capacity of bronchodilator response measured with three different lung function techniques in asthmatic and healthy children aged 2 to 5 years. Am J Respir Crit Care Med. 2001;164(4):554-9. 